\title{
Nutrición saludable: suplementos dietarios en Argentina
}

Prof. Cesar A. Lezcano ${ }^{1}$; Prof. Eva G. S. Miño²; Prof. Lorena Obregon³; Dra. Melisa J. Hidalgo ${ }^{1^{*}}$; Prof. Roberto G. Pellerano ${ }^{1}$

\section{RESUMEN}

Los suplementos dietarios son alimentos destinados a suplementar la incorporación de nutrientes en personas sanas que no se encuentran en estado patológico, siendo la administración de los mismos por vía oral y bajo la supervisión de un profesional de la salud. Este proyecto busca generar la reflexión junto con alumnos del nivel medio, sobre la legislación vigente de dichos productos. Abordando la problemática del consumo indiscriminado y sin supervisión de estos productos, que usados inadecuadamente pueden provocar daños a la salud. Además, se busca difundir los beneficios de una adecuada alimentación, equilibrada y variada acorde a las necesidades de cada persona, para de esa manera evitar la suplementación de la dieta mediante el uso estos productos alimenticios.

\section{PALABRAS CLAVES}

alimentos; educación alimentaria; nutrición saludable

\section{INTRODUCCION}

Los suplementos dietarios (SD) son definidos en el artículo 1381 del Código Alimentario Argentino (CAA), como "productos destinados a incrementar la ingesta dietaria habitual, suplementando la incorporación de nutrientes en la dieta de las personas sanas que, no encontrándose en condiciones patológicas, presenten necesidades básicas dietarias no satisfechas o mayores a las habituales". Los SD comprenden vitaminas, minerales, aminoácidos, glúcidos, proteínas, lípidos, fibras dietarias, en forma simple o combinada. Esta permitida la incorporación de hierbas naturales debiendo indicarse en el listado de ingredientes, indi-

\footnotetext{
(1) Departamento de química, Facultad de Ciencias Exactas y Naturales y Agrimensura, UNNE, Avenida Libertad 5470, Corrientes, Argentina.

(2) Colegio Secundario “Dr. Luis Federico Leloir”, Avenida La Paz 2735, Corrientes, Argentina.

(3) Colegio Secundario Colonia Llano, Ruta Provincial N 4, San Luis del Palmar, Corrientes, Argentina.

* E-mail: melujaz@gmail.com
} 
cando su denominación común, su nombre científico, indicando también la parte somática de la planta. Podrán utilizarse solo las hierbas que se encuentran aprobadas en el CAA.

Se debe tener presente que, en condiciones normales, una dieta habitual debe proveer todos los nutrientes necesarios para poder mantener las funciones del organismo. Los SD están elaborados para complementar las dietas de algunas personas, pero no para reemplazar el equilibrio de los nutrientes presentes en una buena alimentacion. La dieta habitual de las personas debe ser balanceada, variada y moderada, teniendo en cuenta el gasto de energía que tenga cada individuo. Por ende, un SD solo debe consumirse cuando a través de una previa consulta con un profesional de la salud, la persona necesite un aporte extra de uno o más nutrientes.

Los SD son productos que tienen gran aceptación en el mercado, ofreciéndolos en diferentes medios y quienes lo comercializan no siempre of recen información adecuada en cuanto a su manejo, dosificación o contraindicaciones. Adicionalmente, las personas tienden a consumir SD sin previa consulta con su médico o sin realizarse un control de su estado de salud. Por lo tanto, resulta necesario crear conciencia entre los usuarios respecto al uso correcto de estos productos, evitando efectos perjudiciales para su salud. Uno de los objetivos de este proyecto es conocer y estudiar el consumo de los SD por parte de los jóvenes teniendo en cuenta la problemática existente respecto a estos alimentos como ser el desconocimiento de las contraindicaciones y posibles efectos secundarios, ya sea por el consumo de grandes dosis o por combinaciones con fármacos. Para ello se llevaron a cabo actividades en las cuales interaccionaron alumnos de la carrera de Bioquímica de la Facultad de Ciencias Exactas y Naturales y Agrimensura (FaCENA - UNNE) y distintos integrantes de la comunidad educativa, principalmente los alumnos del nivel medio de la provincia de Corrientes. Los destinatarios de este proyecto fueron los integrantes de la comunidad educativa del nivel medio de distintos colegios ubicados uno en el ejido urbano de la ciudad de Corrientes (Capital) y otro en la zona rural correspondiente a la misma. Se realizaron en primer lugar encuestas a fin de determinar el nivel de conocimiento que tienen los alumnos de los diferentes niveles, y a partir de ahí se generó material de difusión (folletos, material multimedia y seminarios) para proveer información y crear conciencia sobre la importancia de una nutrición saludable.

\section{METODOLOGIA}

La difusión y concientización con respecto al uso correcto de los SD disponibles comercialmente en la Argentina es uno de los principales objetivos de este proyecto. Así se intenta brindar herramientas que permitan a los jóvenes y su entorno social mejorar su calidad de vida. Para lograr este objetivo, se realizó 
un monitoreo del nivel de conocimiento que se tenía respecto al tema de los SD, para ello los docentes y alumnos universitarios integrantes del proyecto elaboraron una encuesta. La encuesta anónima contempló datos personales (edad, sexo) e incluía preguntas sobre aspectos relacionados a los $\mathrm{SD}$, opinión y niveles de consumo.

El estudio fue desarrollado en dos establecimientos educativos de nivel secundario de la provincia de Corrientes durante el año 2018. Uno de los colegios se encuentra ubicado en un importante barrio la ciudad de Capital de Corrientes, ubicado en una zona que presenta alta densidad poblacional. E1 otro colegio en la zona rural de la localidad de San Luis del Palmar, a unos $22 \mathrm{~km}$ de la ciudad de Corrientes. Las encuestas fueron realizadas a un total de 70 alumnos, entre 15 y 19 años, en el establecimiento de $\mathrm{Ca}$ pital (CAP) y 195 estudiantes, entre 12 y 22 años, en el establecimiento de San Luis del Palmar (SLP). En ambas instituciones del total de encuestados, aproximadamente el $60 \%$ fueron mujeres y el $40 \%$ restante fueron varones. Las encuestas fueron entregadas por los docentes co-participantes del proyecto y alumnos colaboradores. La participación de los alumnos en la encuesta fue voluntaria.

Los resultados obtenidos se procesaron a través de programa de planilla de cálculos. Estos resultados nos permiten conocer y comparar la prevalencia de consumo de los SD de una determinada franja etaria. Proporcionando evidencia que contribuirá a formular replanteamientos sobre la temática SD.

\section{RESULTADOS Y DISCUSION}

A partir del procesamiento y análisis de los datos obtenidos de las 265 encuestas realizadas en dos establecimientos de nivel medio a los alumnos entre 12 y 22 años que participaron del proyecto se desprende algunos aportes interesantes para este trabajo. Las figuras 1 y 2 muestran de manera gráfica los resultados teniendo en cuenta la forma de presentación comercial de los SD, los resultados obtenidos fueron similares en proporción independientemente de la zona de influencia de cada escuela. Los alumnos mayoritariamente consideran que los SD vienen en presentaciones tales como cápsulas seguido por aquellos que considera que se presentan en forma de bebidas. Sin embargo, no deja de resultar preocupante el hecho de que en ambas instituciones aproximadamente un $6 \%$ de los encuestados respondió que los SD pueden presentarse comercialmente en forma de inyecciones. Más allá de que la respuesta es incorrecta, genera preocupación debido a que los alumnos podrían adquirir ciertos productos bajo esta presentación considerando que son SD y no medicamentos, además del riesgo que implica el uso incorrecto de la manipulación de inyecciones. E1 CAA establece que los SD deben ser de administración oral, se pueden comercializar en forma de comprimidos, cápsulas, tabletas, líquidos o polvos u otras formas para su absorción gastrointestinal. 


\section{FORMA DE PRESENTACIÓN DE LOS SD - CAP.}

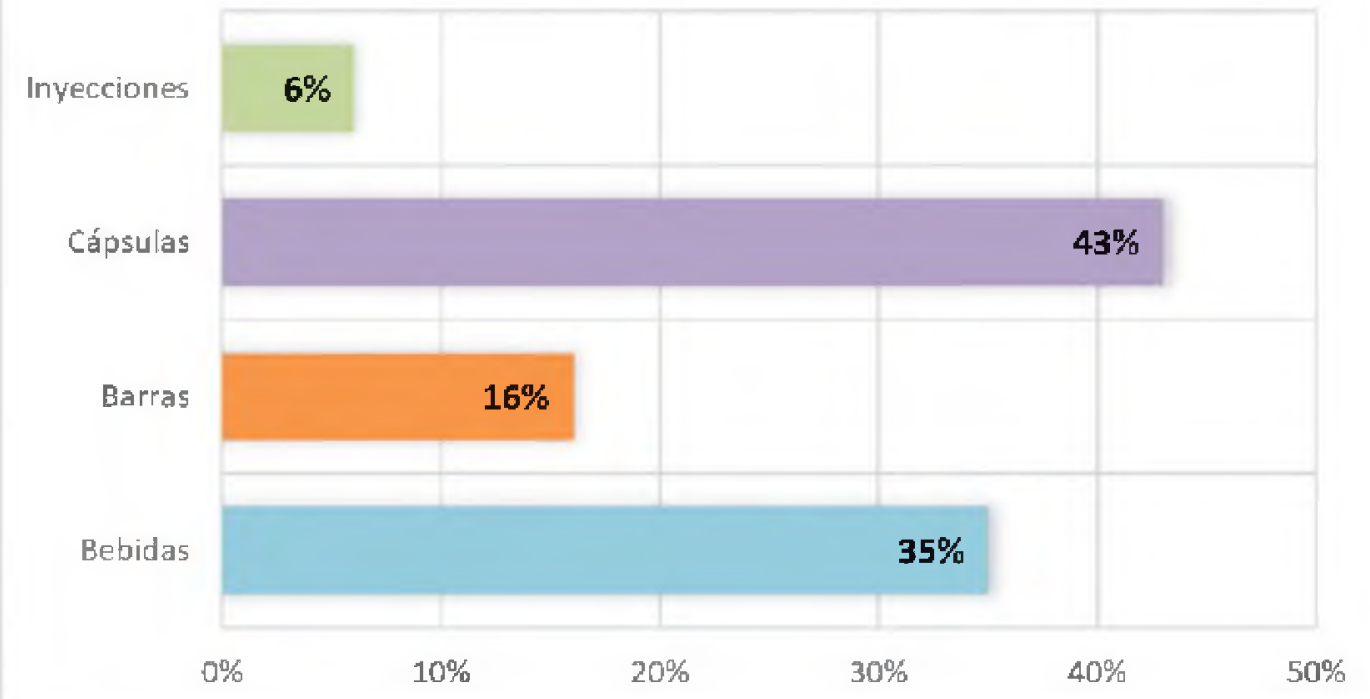

Figura 1. Respuestas obtenidas en el establecimiento de Capital, al preguntar sobre las formas de presentaciones comerciales de los SD.

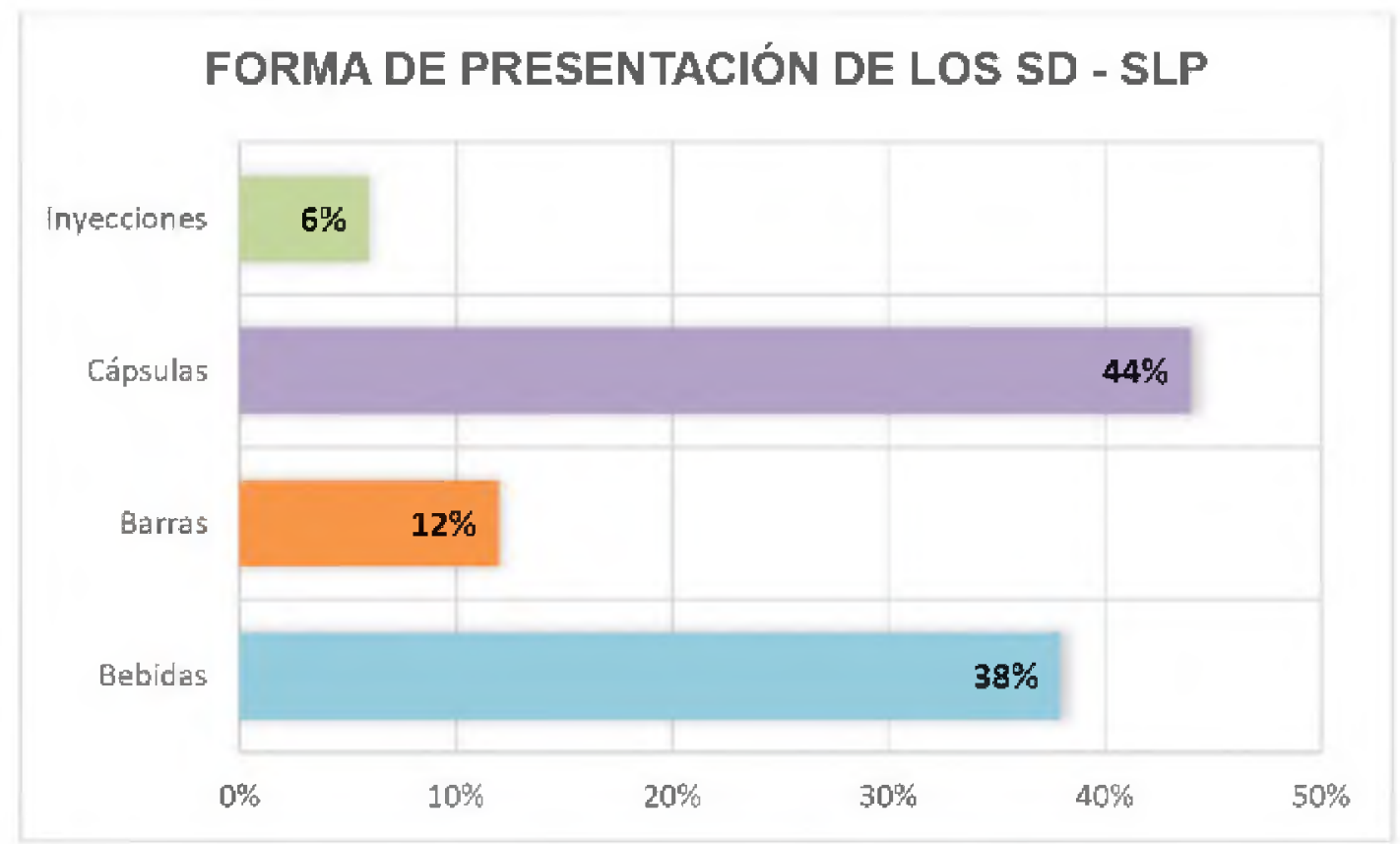

Figura 2. Respuestas obtenidas en el establecimiento de San Luis del Palmar, al preguntar sobre las formas de presentaciones comerciales de los SD. 
En cuanto a la función de los SD (Fig. señalar que los SD no reemplazan el hecho 3 y Fig. 4), los alumnos independientemen- de llevar a cabo una dieta saludable y vate de su procedencia respondieron que los riada acorde a las necesidades nutricionales SD se utilizan para cubrir las falencias en de cada individuo. Este tipo de respuestas los requerimientos energéticos, para rea- plantea la necesidad de acercar información lizar actividad física y para complementar precisa, y transmitir a la comunidad sobre dietas destinadas al control del peso. La las funciones de estos productos, a fin de principal diferencia se encontró en que evitar riesgos para la salud en forma indivilos alumnos de CTE respondieron en una dual o colectiva. Los SD tienen por objeproporción mayor que los SD pueden uti- tivo garantizar la suplementación adecuada lizarse para sustituir una buena dieta. Con de uno o más nutrientes, a fin de reducir el respecto a esta respuesta, resulta importante riesgo de padecer enfermedades.
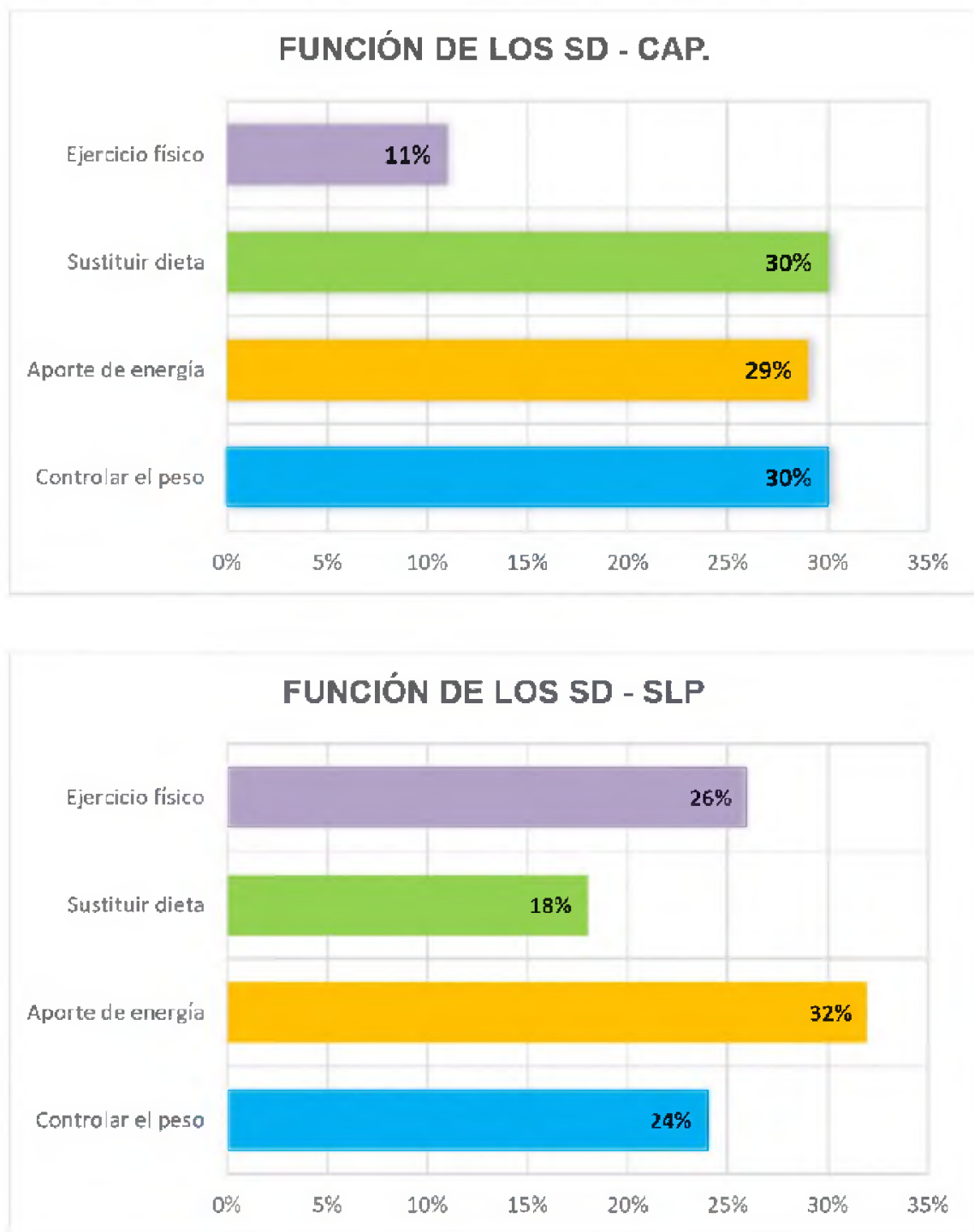

Figura 3 .

Respuestas

obtenidas en la escuela de Capital, al preguntar sobre las funciones que tienen los SD.
Figura 4.

Respuestas

obtenidas en la escuela de San Luis del Palmar, al preguntar sobre las funciones que tienen los SD. 
En consideración a los lugares donde bilidad en supermercados, posiblemente pueden adquirirse o comprarse los SD, debido a las diferencias entre las ofertas los resultados muestran que son adquiri- comerciales disponibles en cada zona. La dos en farmacias principalmente, aunque presencia de los SD en farmacias podría los SD no son medicamentos, seguido por deberse a que la comercialización princisupermercados y kioscos. Se debe des- pal de estos productos se realiza a través tacar, que las respuestas de los alumnos de las empresas farmacéuticas, y en mede las dos escuelas encuestadas variaron nor medida de las empresas alimenticias sensiblemente con respecto a la disponi- (Fig. 5 y Fig. 6).

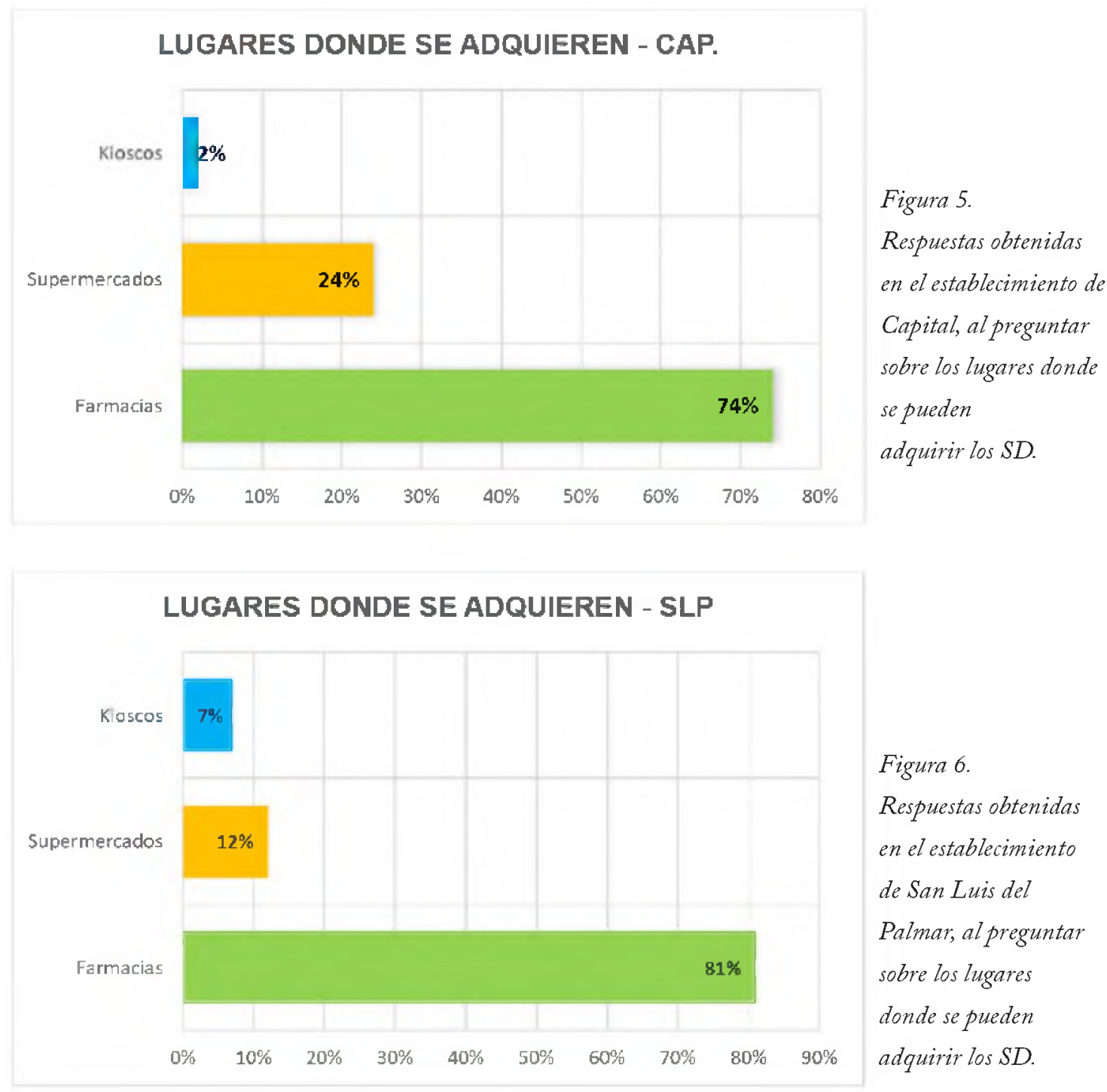



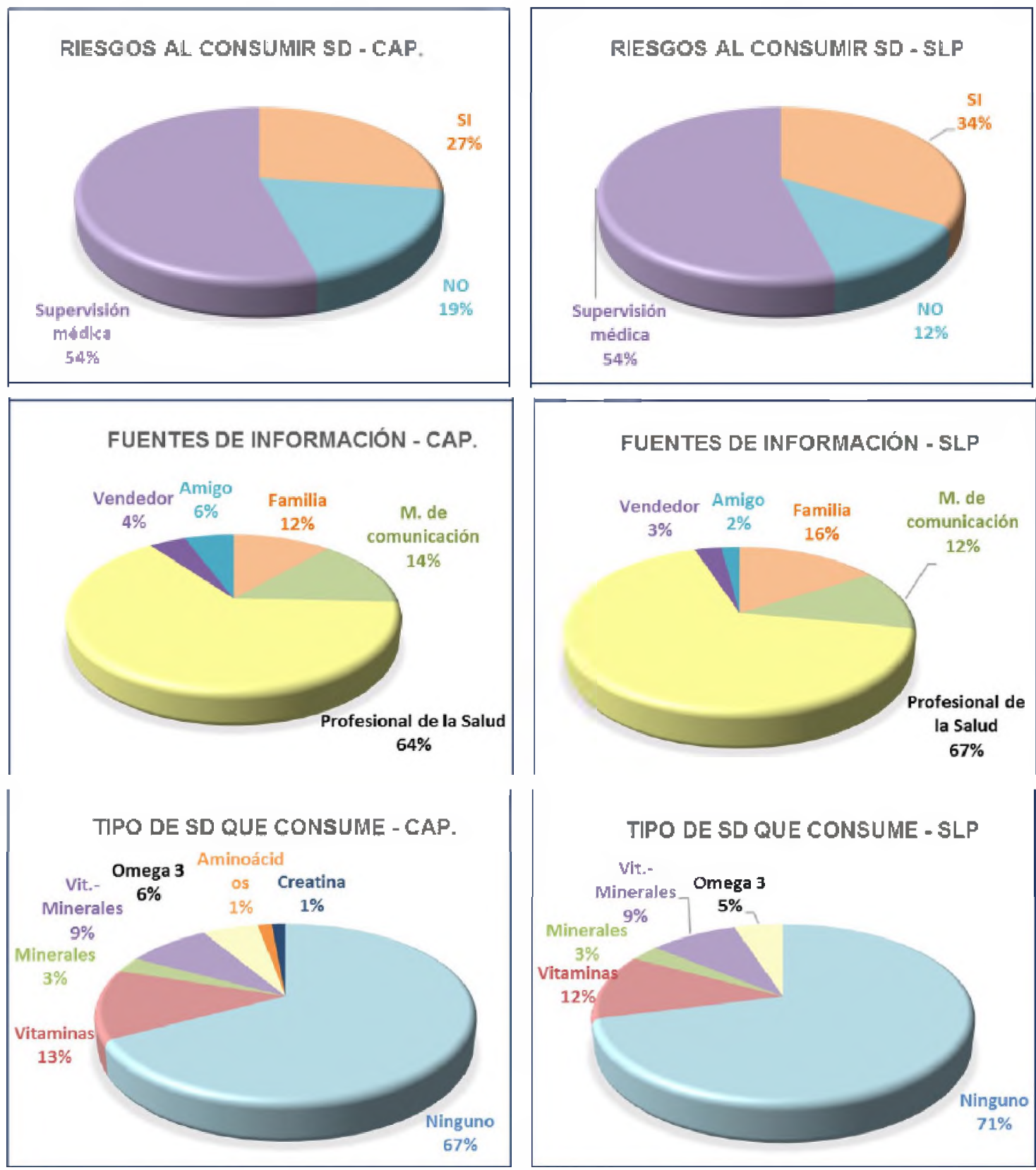

Figura 7. Respuestas obtenidas en ambos establecimientos, al preguntar sobre los riesgos,

fuentes de información y tipo de SD que consumen.

Finalmente, las encuestas incluyeron tres preguntas similares a las publicadas en el año 2016 (LUTZ, et al.) en el colegio secundario de CAP. En relación con el riesgo que existe el que necesita de la supervisión de un médico consumo de $\mathrm{SD}$, en ambos establecimientos encuestados más del 25\% considera que el consumo de estos productos presenta riesgos para la salud y más del 50\% reconoce 
para la administración adecuada de estos alimentos (Figura 7). Por otro lado, en 2016 solo un $10 \%$ consideraba que el consumo de SD representaba algunos riesgos para la salud, mientras que el $67 \%$ consideraba que si había riesgos para la salud y por lo tanto necesitaban de la supervisión de un médico.

Las respuestas de los alumnos frente a la consulta sobre cual es la fuente principal de información que tienen sobre los $\mathrm{SD}$, en ambos establecimientos más del $60 \%$ obtenía la información de un profesional de la salud. En segundo lugar, en el colegio de CAP. siguen los medios de comunicación, a través de la publicidad principalmente, mientras que en el colegio de SLP obtenían información de la familia (Figura 7). Este último esquema de respuestas obtenido de la escuela de SLP es similar al encontrado en la escuela de CAP. en el 2016. En cuanto a la publicidad de estos alimentos, la Administración Nacional de Medicamentos, Alimentos y Tecnología Médica (ANMAT) fija pautas éticas, algunas de ellas hacen referencia a que no deberán incluir frases o mensajes que atribuyan propiedades o acciones terapéuticas; manifiesten que pueden ser usados en reemplazo de una comida convencional, entre otros. Además, la ANMAT fiscaliza la publicidad de dichos alimentos a fin de reducir riesgos para los consumidores.

Los alumnos respondieron también acerca de que tipos de SD consumían, más del 60\% de los alumnos de CAP, declaró no consumir ningún tipo al igual que más del $70 \%$ de los alumnos de SLP. En cuanto a los que ingieren algún tipo de SD, más del 10\% en ambos colegios consumen vitaminas, seguido por combinaciones de vitaminas $y$ minerales. Algo similar a lo observado a los encuestados en 2016, en la cual también algunos alumnos reconocían el consumo de creatina con fines deportivos. Se debe tener en cuenta que las vitaminas y minerales propuestos para la suplementación de la dieta deberán cubrir no menos del 30\% de la Ingesta Diaria Recomendada (IDR) de acuerdo con los valores que figuran en el CAA en el artículo 1387. En la encuesta no fue incluida la pregunta respecto a que tipo de vitaminas o minerales consumían (Figura 7).

Finalmente, se debe destacar que los jóvenes no deben consumir SD por moda o tendencias ni con la esperanza de aliviar alguna dolencia o patología. Solo deberán consumirlos cuando, por un estado fisiológico particular, necesiten un aporte extra de algún tipo de nutriente, previa consulta con un profesional de la salud.

\section{CONCLUSIONES}

A partir de los resultados obtenidos en este trabajo a través de las encuestas realizadas a los alumnos de los dos establecimientos de nivel medio, uno en la ciudad de Corrientes y el otro en la localidad próxima de San Luis del Palmar surge prioritariamente la necesidad de incorporar y actualizar la información respecto a los $\mathrm{SD}$, a fin de educar y concientizar a la comunidad en el buen uso y adquisición de estos. En base a los resultados obtenidos en las encuestas, los estudiantes universitarios junto con 
docentes de la carrera de Bioquímica de la rios brindados en los colegios secundarios FaCENA integrantes del proyecto "Nutri- para los distintos integrantes de la comución Saludable: Suplementos Dietarios en nidad educativa. De esta manera a modo Argentina" en colaboración con alumnos y de estrategia de sensibilización, se propuso docentes de las dos escuelas de nivel medio fomentar el conocimiento respecto a estos co-participantes, elaboraron material de di- alimentos, evitando su uso indebido a trafusión (folletos, cartelería y material multi- vés del asesoramiento de profesionales de la media) que fueron difundidos en semina- salud capacitados.

\section{BIBLIOGRAFIA}

ANMAT. Código Alimentario Argentino. Capítulo XVII. http://www. anmat. gov. ar/alimentos/codigoa/CAPITULO_XVII (Accedido 23/11/2018).

Lutz, C. C., Fernandez, M. F., Lezcano, C. A., Moresi, A. L., Goyechea, R.I., Fechner, D. C., Miño, E. G. S., Esquivel, M., Ruiz Diaz,
J. D., Pellerano, R. G. Suplementos Dietarios: ¿Cuánto sabemos para poder usarlos correctamente? Extensionismo, Innovación y Transferencia Tecnológica, Claves para el Desarrollo, (3), 214-218. (2016).

U.S. Food and Drug Administration (FDA). Dietarysupplements. http://www.fda.gov/ Food/DietarySupplements/

(Accedido 22/11/2018). 